\title{
Fecal Occult Blood Test
}

National Cancer Institute

\section{Source}

National Cancer Institute. Fecal Occult Blood Test. NCI Thesaurus. Code C18014.

A noninvasive test that detects the presence of hidden (occult) blood in the stool. This is commonly performed to screen for colon cancer. 